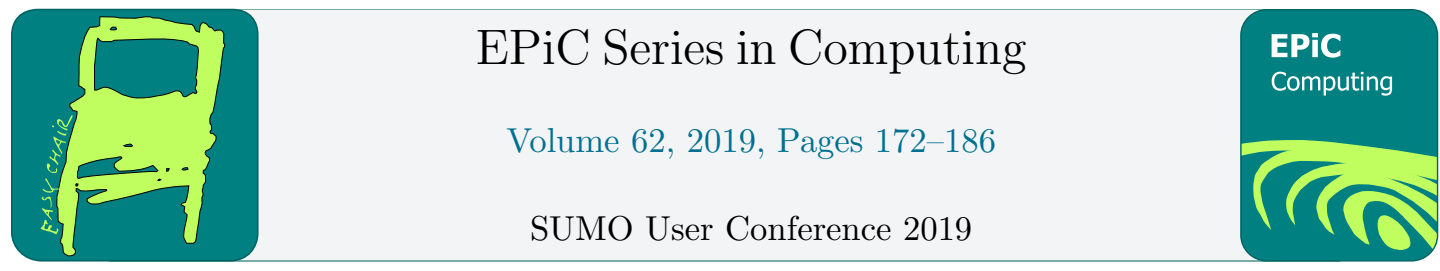

\title{
Introducing Road Surface Conditions into a Microscopic Traffic Simulation
}

\author{
Thomas Weber ${ }^{1}$, Patrick Driesch ${ }^{1}$, and Dieter Schramm ${ }^{1}$ \\ ${ }^{1}$ University Duisburg-Essen, Chair of Mechatronics, Duisburg \\ \{thomas.markus.weber, patrick.driesch, dieter.schramm\}@uni-due.de
}

\begin{abstract}
The introduction of highly automated driving functions is one of the main research and development efforts in the automotive industry worldwide. In the early stages of the development process, suppliers and manufacturers often wonder whether and to what extend the potential of the systems under development can be estimated in a cheap and timely manner. In the context of a current research project, a sensor system for the detection of the road surface condition is to be developed and it is to be investigated how such a system can be used to improve higher level driving functions. This paper presents how road surface conditions are introduced in various elements of the microscopic traffic simulation such as the actual network, the network editor, a device for detection, and an adaptation of the standard Krauß car following model. It is also shown how the adaptations can subsequently affect traffic scenarios. Furthermore, a summary is given how this preliminary work integrates into the larger scope of using SUMO as a tool in the process of analyzing the effectiveness of a road surface condition sensor.
\end{abstract}

\section{Introduction}

The development of highly automated and autonomous driving functions comes with new requirements regarding compliance with safety in all situations. Present advanced driver assistance systems (ADAS) merely support the driver in his task, whereas all decision-making responsibilities remain with him. In the context of autonomous driving however, these responsibilities will lay with the system as well. 
While it is true that computer systems do not get tired or distracted and have the potential to react faster than a human driver [2], they can only act upon the input data available. Thus, in order to arrive at reasonable and reliable decisions, a multitude of sensor and environmental data is required. Since there is no commercially available automotive sensor to detect the effective road surface condition, it is the goal of a current research project called Seeroad [3] to develop a new sensor device for this purpose to enable current ADAS the transition to highly automated driving functions.

In the early stage of development however, it is difficult to determine the potential of the new system not only towards a single given driving situation, but also on a broader scale such as traffic safety, traffic flow and traffic efficiency. Since prototypes that may be used in real vehicles for testing are only available in later product development stages and can usually only be used to assert certain functions or driving situations, simulations and especially traffic simulations are a cheap and efficient way to answer a variety of research questions.

Within the Seeroad project, one of the approaches followed to evaluate possible impacts of the sensor system in development on various levels of complexity is shown in Figure 1.

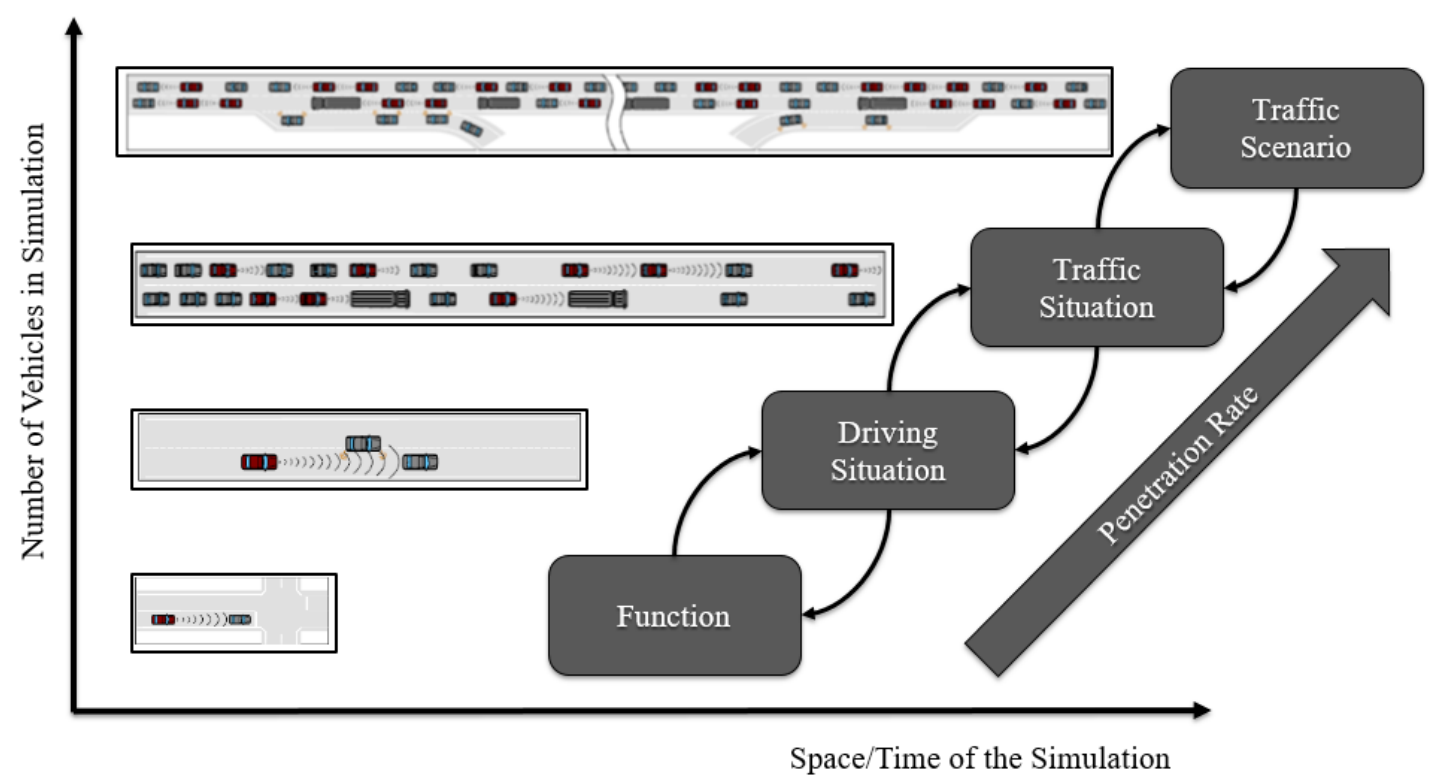

Figure 1: Assessment approach of new functions in the driving environment in accordance with [4]

This paper will focus on the integration of road surface conditions in the upper levels of the investigation plane, which deal with traffic situation and complex traffic scenarios. As traffic simulation environment the open source framework SUMO is used. It has proven its capability in traffic research on a wide range of different topics [5] and offers the possibility of being augmented to the specific needs of the 
investigation approach. Using real-world calibrated traffic scenarios as a reference, it is possible to analyze the changes in certain relevant key values when introducing novel systems and derive assessments about their potential influence by interpreting the data accordingly.

In Section 2 an overview of the implementations made to incorporate road surface conditions into the framework is given. Section 3 shows the result of an example simulation utilizing this new feature with emphasis on its effectiveness towards the car following model. Finally, Section 4 summarizes the findings and gives an outlook on future works that will be done with this new implementation.

\section{Implementation}

In order to implement road surface conditions into SUMO and make it accessible to the mathematical models of the program it is necessary to identify a way to describe these as a numerical value. In the automotive context, the road surface condition is one of the main influencing factors on the coefficient of friction between the tire and the road surface. [1] [6] The friction value has influence on the transmittable longitudinal and lateral forces between a vehicle and the

\begin{tabular}{ll}
$\begin{array}{l}\text { Surface } \\
\text { Condition }\end{array}$ & $\begin{array}{l}\text { Road Friction } \\
\text { Coefficient }\end{array}$ \\
\hline Dry & $\mu \approx 1.0-1.2$ \\
\hline Moist & $\mu \approx 0.8-1.0$ \\
\hline Wet & $\mu \approx 0.5-0.8$ \\
\hline Snow & $\mu \approx 0.3-0.6$ \\
\hline Ice & $\mu \approx 0.1-0.4$ \\
\hline
\end{tabular}

Table 1: Relation between road surface conditions and its friction coefficient [1] road and thereby also on the stability of the driving process. Table 1 shows how different road surface conditions affect the values of the friction coefficient. Since a well-known single numerical value with relation to the road surface condition exists, it is consistent to introduce this value as an additional attribute of the edge and lane elements of the network used in SUMO, as they represent the roads. To ensure backward compatibility, a default value of 1.0 is set upon parsing when using network xml files without specified friction values. A specific value can be set within these $\mathrm{xml}$ files like other attributes through the key value pair friction=" $\angle$ DOUBLE $>$ " within the respective tags. It is defined as a dynamic attribute and the implementation is as such that the value can be changed online while the simulation is running either through the TraCI (Traffic Control Interface) or via a trigger object that can be specified within an additional file. 
造 circles.net.xml - NETEDIT 0.32.1

UI

\section{Net: edge}
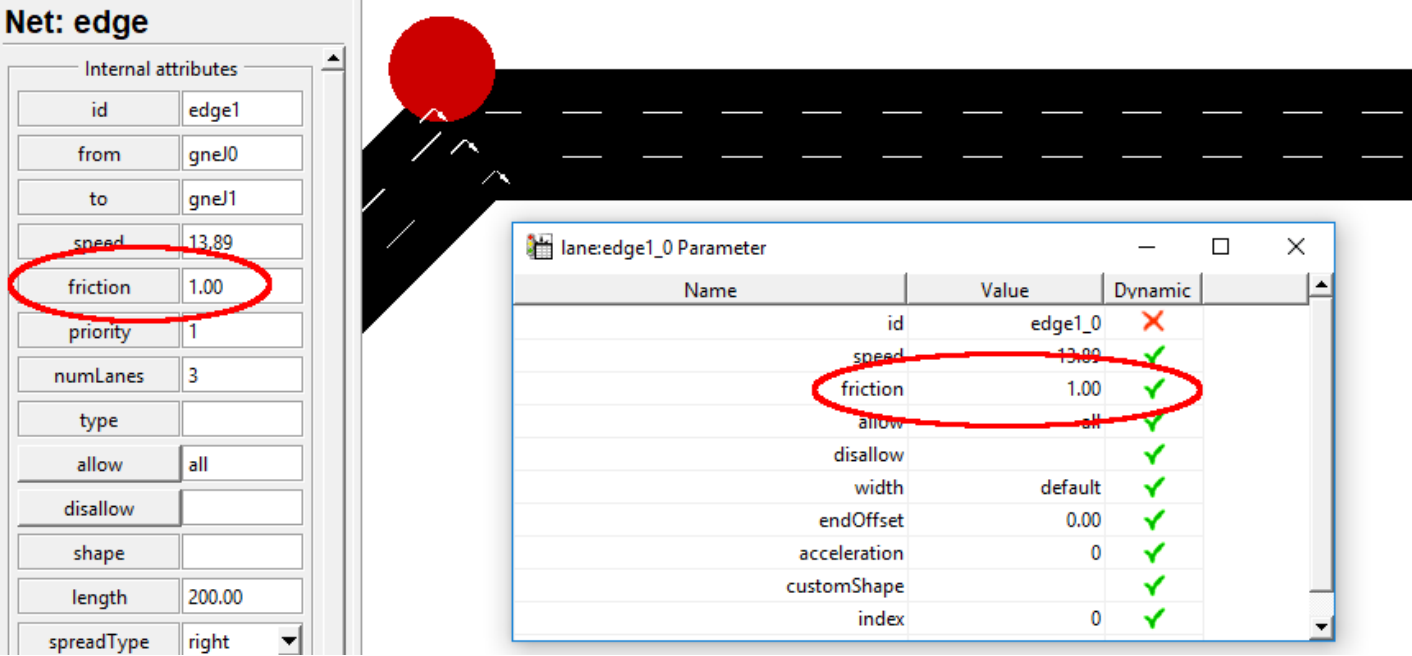

Figure 2: Friction Coefficient Attribute as seen in NETEDIT

While the direct manipulation of network files might be feasible for very small networks with only few edges and a very limited number of lanes, it becomes very tedious for larger scenarios quickly. Therefore NETEDIT, SUMOs graphical network editor, is also amended to accommodate and manipulate the new attribute as shown in Figure 2.

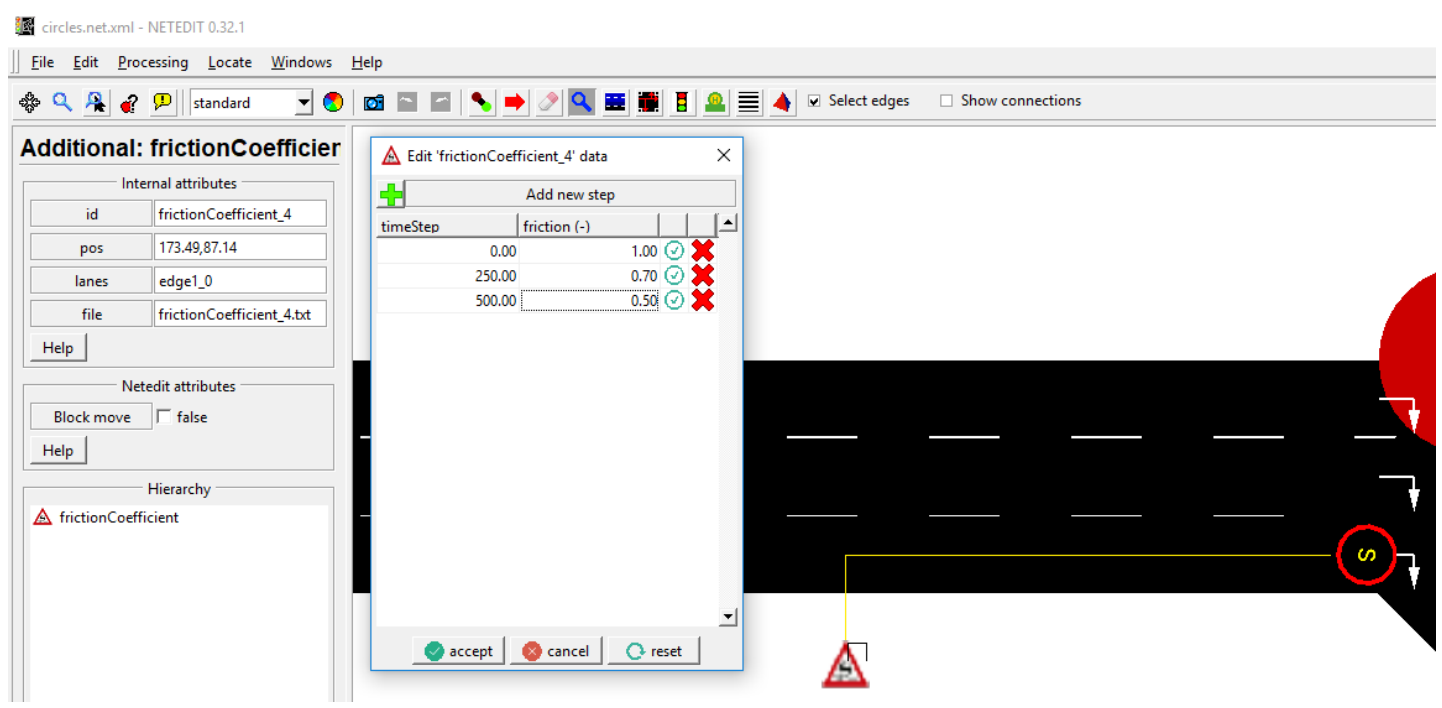

Figure 3: Friction Coefficient Trigger Object 
The former mentioned trigger object for the online manipulation can be found in the additional menu and placed upon the desired lanes. The trigger object allows for online manipulation of the friction value either using the SUMO-GUI, the TraCI interface or by scheduling changes at certain time steps within an additional xml file. These can be set through the NETEDIT GUI as shown in Figure 3 and will automatically outputted in the necessary xml format as shown in Listing 1 . The listing demonstrates also how to switch the friction coefficient of the road surface dynamically at specific points of time during the simulation.

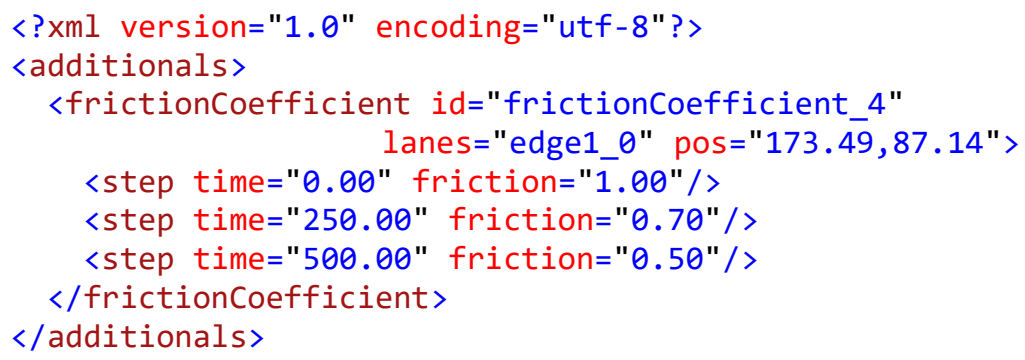

\section{Listing 1: XML output of the additional trigger object}

Thus far the implementation covered the introduction of the road surface condition as a property of the simulation network. The next step in the implementation process is to enable individual vehicles in the simulation to pick up and interact with this attribute in the manner of its intended reflection of a road surface condition sensor. For purposes like this, SUMO has a designated class called a "device" which may be equipped to individual vehicles or a class of vehicles. In this case a new device called "MSDevice_Seeroad" is derived from that class. The Seeroad device is designed to simulate the sensor device and to be able to hold various mathematical models if necessary. Since there is not enough usable real-world measurement data from the emerging prototype in order to implement a complex model at the time of this initial implementation, the actual model simply consists of uniformly distributed measurement noise. The thus created simulated measurement data output is added to the existing floating car data (FCD) output format. The implementation is such that the friction key value pair appears within the attribute list of the output tags only of those vehicles that are in fact equipped with a Seeroad device. Figure 4 depicts an example for the respective output of a single Seeroad device equipped vehicle for a given friction coefficient of 1 . 


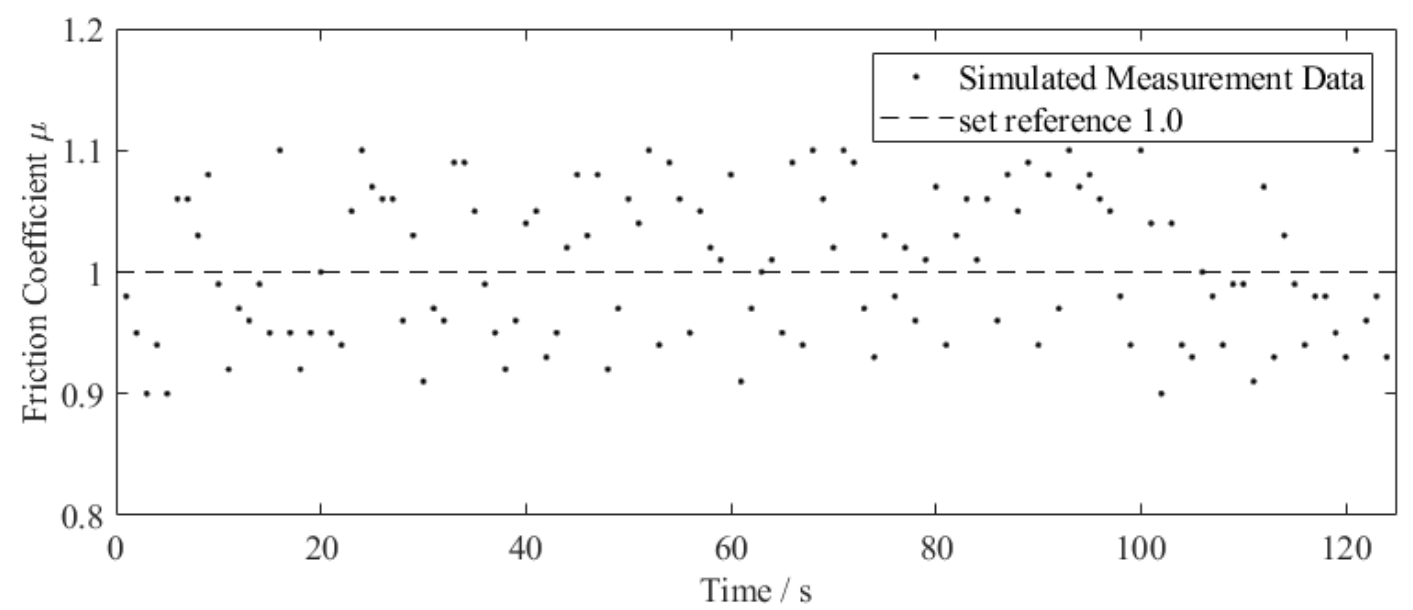

Figure 4: Example Seeroad device output for a single equipped Vehicle

Furthermore, TraCI is adapted to fully support online access to the actual measurement data point through the selective generic parameter retrieval call as well as through subscriptions to the respective vehicle variable. This becomes necessary when using the data in a larger co-simulation environment. One premise for this preliminary work is to analyze the impact and potential not only on present day traffic scenarios, but also with respect to emerging trends. One of these is the growing connectivity of vehicles. In order to cover this area the Veins [7] framework is used, which uses TraCI to connect a network simulation performed by OMNeT++ [8] to the traffic simulation SUMO while using the vehicles as nodes. The adaptations to $\mathrm{TraCI}$ make it possible to access all aspects of the road surface condition itself as well as the sensor device.

To satisfy the before mentioned approach to use a realistic base traffic scenario as a reference to describe and estimate the influence of road surface condition aware systems in future scenarios, it is necessary to adapt a driver model within the simulation to emulate a realistic driver behavior under adverse road surface conditions.

Some studies regarding the influence of weather-related road surface conditions on traffic can be found in the literature. Table 2 gives an overview of some relevant results of such research and it becomes clear that the road condition has a significant influence on the observed speeds in the traffic flow. It should be noted that this is only a general summary of the final results and that no further subdivision of the data is made here. The reasons for the range of the results are the fact that the studies on the one hand take the severity of the precipitation into account and various types of measuring stations and different road types on the other. Another reason for the large variation is the overlapping of different additional effects, such as twilight, darkness or other visual impairments for example. 


\begin{tabular}{|l|c|c|c|c|}
\hline $\begin{array}{l}\text { Weather } \\
\text { (Surface) } \\
\text { Condition }\end{array}$ & Agarwal [9] & Stern [10] & HCM [11] & Goodwin [12] \\
\hline $\begin{array}{l}\text { Rain (wet) } \\
\text { Speed reduction: }\end{array}$ & $2-17 \%$ & $5-25 \%$ & $0-15 \%$ & $10-25 \%$ \\
\hline $\begin{array}{l}\text { Snow (slippery) } \\
\text { Speed reduction: }\end{array}$ & $10-27 \%$ & $12-27 \%$ & $10-35 \%$ & $13-40 \%$ \\
\hline
\end{tabular}

Table 2: Summary of research of weather impact on traffic speeds

However, together with the information from Table 1, the relevant findings of these researches in terms of observed speed reductions in different forms of precipitation are used to estimate a model relationship between the condition of the road surface and the reduction of speed. Figure 5 shows two possible models based on a first and second degree polynomial fitting of the data derived from the findings summarized in Table 2 and correlated to the range values found in Table 1.

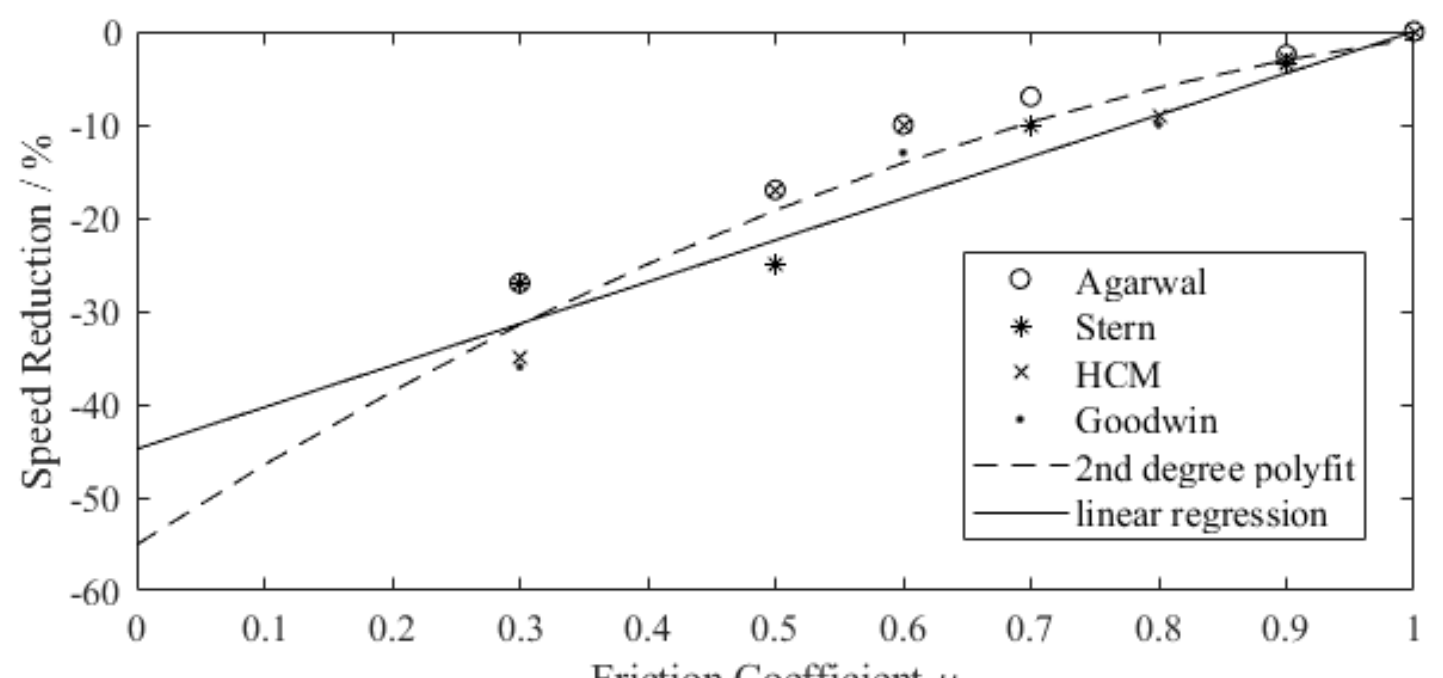

Figure 5: Speed reduction model for the car follow model adaptation

According to the linear model, a wet road, described by the range $\mu \approx 0.5-1.0$, leads to a speed reduction of $0-22.4 \%$ and a snowy road, represented by the friction value range $\mu \approx 0.3-0.6$, leads to a reduction of $17.9-31.4 \%$. While with the quadratic model a speed reduction within the wet road range of $1-19.2 \%$ and in the snowy road range of $14.1-31.5 \%$ is to be expected. 


$$
\begin{aligned}
& f(\mu)=0.4481 \mu+0.5720 \\
& f(\mu)=-0.3491 \mu^{2}+0.8922 \mu+0.4493
\end{aligned}
$$

Equations (2-1) and (2-2) describe the derived speed reduction factor of the fitted relative speed reduction models. Both equations are implemented into an augmented standard Krauß [13] car follow model in order to more closely simulate a drivers behavior under adverse weather conditions. The implementation ensures, that the model can be adapted easily. The results of all implementations are discussed in the next Section.

\section{Simulation}

To verify the effectiveness of all parts of the implementation, a reference scenario consisting of a motorway section is created and calibrated with traffic data collected from induction loops provided by the German Federal Highway Research Institute (BASt) [14]. A motorway interchange in the vicinity of the city Suhl is used, because several induction loops and their data are available in a relatively small area and this simplifies calibration. Figure 6 depicts the chosen section in form of the OpenStreetMap source as well as the result after its import into NETEDIT and the corresponding placement of induction loops based on the position of their counterparts in the real world.

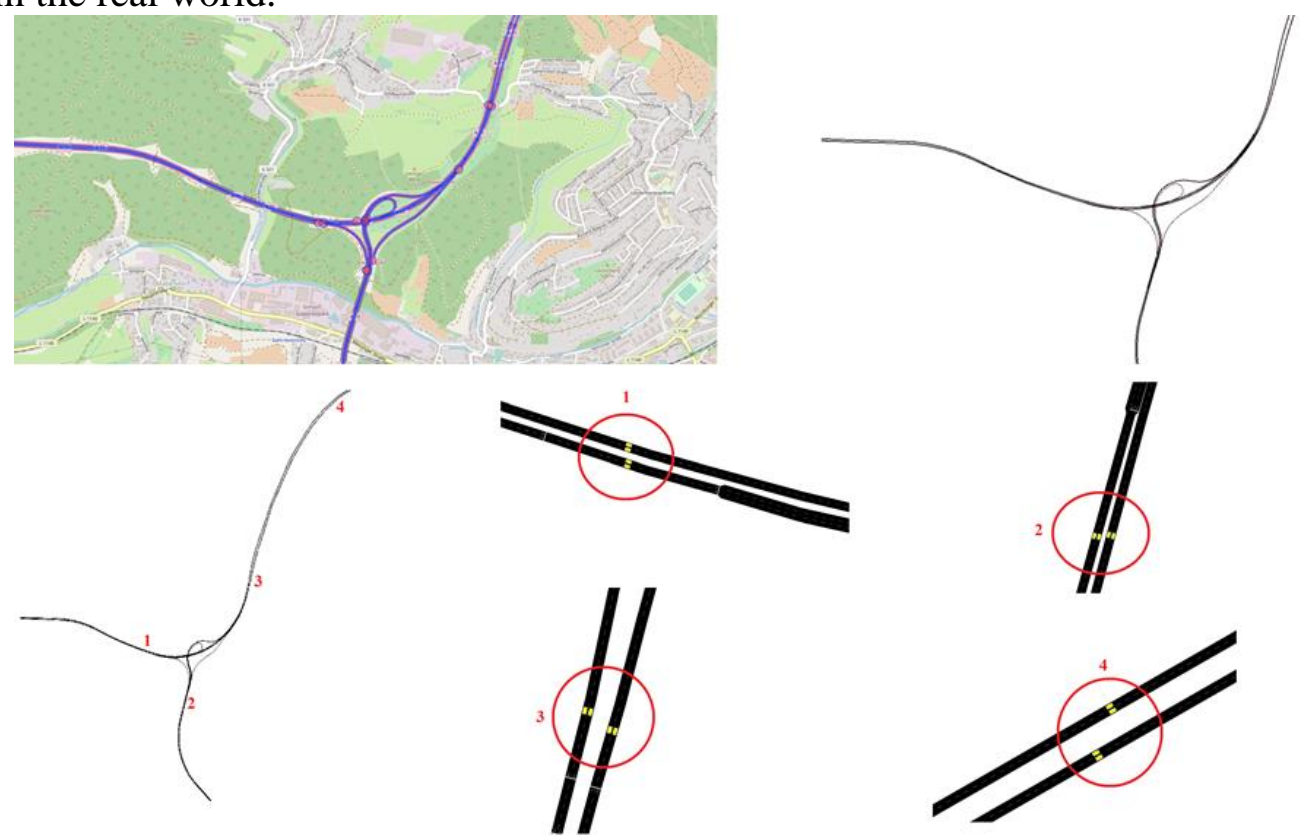

Figure 6: Suhl motorway interchange network and induction loop placement 
The traffic data are available hourly in an aggregated form and describe the macroscopic value of traffic flow $Q$, which is generally defined as the number of vehicles $\Delta N$ that have passed through the cross-section at point $x$ in time interval $\Delta t$. The flow is usually expressed in terms of vehicles per minute or vehicles per hour. [13]

$$
Q(t, x)=\frac{\Delta N}{\Delta t}
$$

The calibration of a reference traffic scenario is a constrained optimization problem. With a motorway network which has detectors at all entrances and exits, it can first be assumed that the sum of all entering vehicles is equal to the sum of the leaving vehicles. At measurement points where vehicles enter the network, there is only one possibility how the measurement data is composed, because at these points all counted vehicles come from the same direction. Counting at network exits becomes more difficult. Here, the number of vehicles consists of all potential routes leading from the entrances to this exit. With this premise it is possible to represent the present optimization problem as a linear equation system with additional requirements to the constraint parameters. In matrix notation a linear system of equations can first be divided into a coefficient matrix $A$, the parameter vector $\boldsymbol{x}$ and a result vector $\boldsymbol{b}$.

$$
\begin{aligned}
& \boldsymbol{A}_{n, k} \boldsymbol{x}_{k, 1}=\boldsymbol{b}_{k, 1} \\
& x_{1} \boldsymbol{a}_{\mathbf{1}}+x_{2} \boldsymbol{a}_{2}+\cdots+x_{k} \boldsymbol{a}_{\boldsymbol{k}}=\boldsymbol{b}
\end{aligned}
$$

In the vector form (3-3) it becomes clear that the system of equations is solvable if the result vector can be represented as a linear combination of the column vectors $\boldsymbol{a}$. In other words, the system of equations can only be solved if the result vector lies in the column space of the coefficient matrix. In real systems, however, it is often the case that measurements are subject to certain perturbations. Due to inexact data in the coefficient matrix or the result vector, it can quickly happen that the result vector no longer lies in the column space of the coefficient matrix and thus no unique solution for the equation system exists. The least squares method is now based on the assumption that there must be one or more linear combinations within the column space that come closest to the result vector. This approach thus minimizes the distance from the result vector to the column space.

$$
\min _{\overrightarrow{\boldsymbol{x}} \in \mathbb{R}^{n}}\|\boldsymbol{b}-\boldsymbol{A} \boldsymbol{x}\|=\sqrt{\sum_{i=1}^{n}\left(b_{i}-\sum_{j=1}^{n} a_{i j} x_{j}\right)^{2}}
$$

All vectors that are perpendicular to column space belong to the zero space of the transposed coefficient matrix, so for the special solution $\boldsymbol{x}^{*}$ must hold:

$$
A^{T}\left(A x^{*}-b\right)=0
$$




$$
A^{T} A x^{*}=A^{T} b
$$

The special solution $\boldsymbol{x}^{*}$ then designates the solution according to the least squares method, since the distance squares are minimized according to equation (3-4). The system of equations according to (3-6) can now be solved with a suitable method for linear systems of equations. In this case the Sequential Least Squares Programming (SLSQP) algorithm [15] is applied. Although the available data allows for a more detailed subdivision in vehicle types, for simplicity, only two classes are used for this proof of concept paper. Those two classes are a passenger car vehicle type and a heavy truck type. The differences are defined by their attributes such as length, acceleration and deceleration capabilities and maximum speeds. Figure 7 shows the summarized result for the generated scenario based on a random excerpt from the real-world induction loop data.

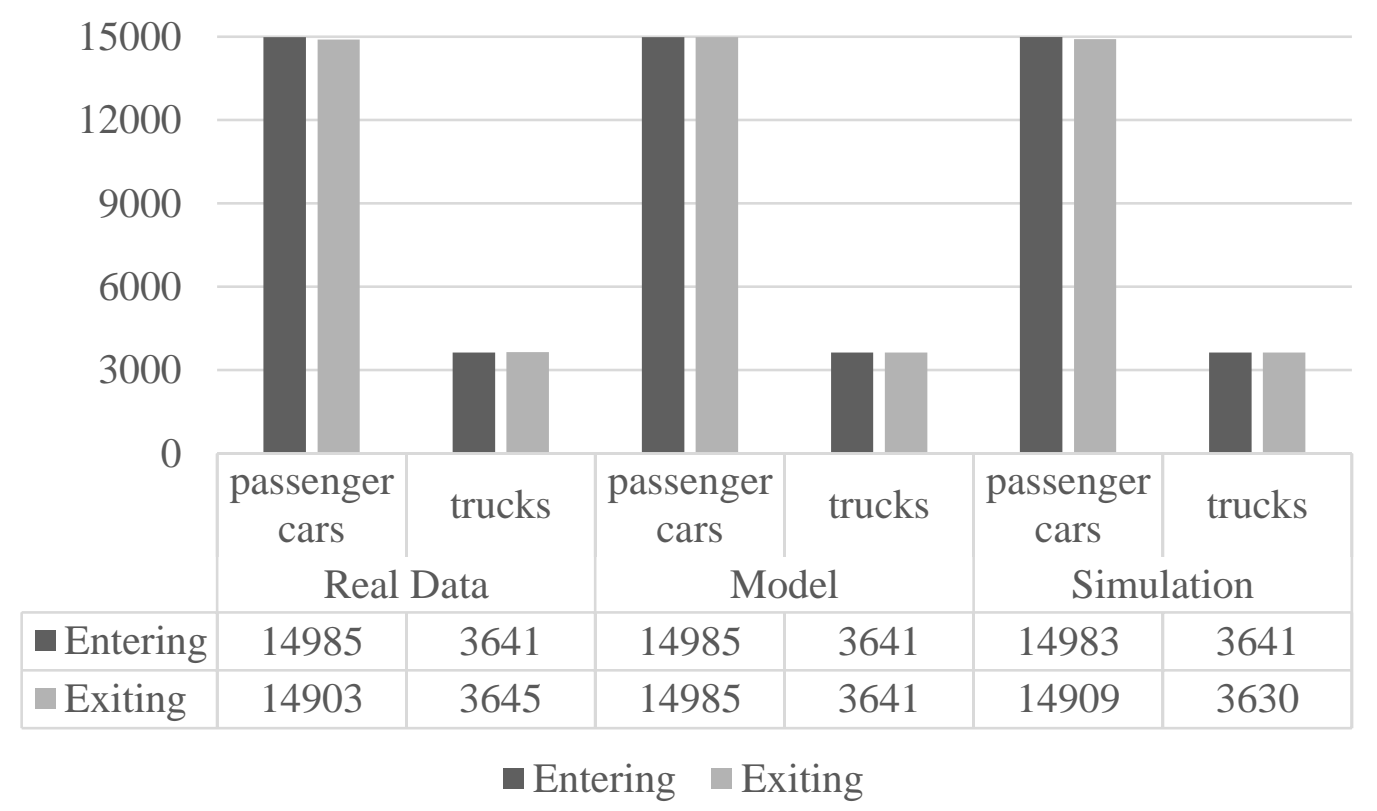

Figure 7: Traffic volume of the motorway scenario

The traffic data are the records from Monday 25.01.2016, aggregated for each hour over the twelve-hour period from 07-19 o'clock. The optimization targets the nodes, i.e. the correct number at each hour, as well as the best overall result with respect to the total numbers. The simulation terminates time-controlled after completing the twelve-hour period, which leads to the difference in entering vs. exiting vehicles within the Scenario. Once the termination criterion is reached, some vehicles have already entered the network and passed the respective detector, but have not yet reached the exit detector. It can be asserted from the results of the simulation that this approach produces an acceptable scenario of a motorway section on at daytime of a 
random weekday. Since the simulation cycles of the traffic adaptation optimization iteration are done with the standard parameterized Krauß car following model, this scenario is now used to verify the effectiveness of the corresponding friction depending adaptation.

For the assessment of the implementation effectiveness of the modified car following model, the scenario is run a total of nine times for each of the two models, with alternating road surface conditions, i.e. friction coefficient values. The average speed for each hour in the scenario is then used as data points to plot and compare with the expectation from the implemented model. Some of the results for a random selection of the 16 detectors is shown in Figure 8, where the black data points represent the average speeds per one hour of observation for each of the simulation runs and the dashed line is the calculated value from equation (2-1) with respect to the reference average speed of the scenario simulation with the unaltered car follow model. It can be stated that the reduction of speed in the simulation based on the given road surface condition follows the intended gradient of the implemented model nicely.

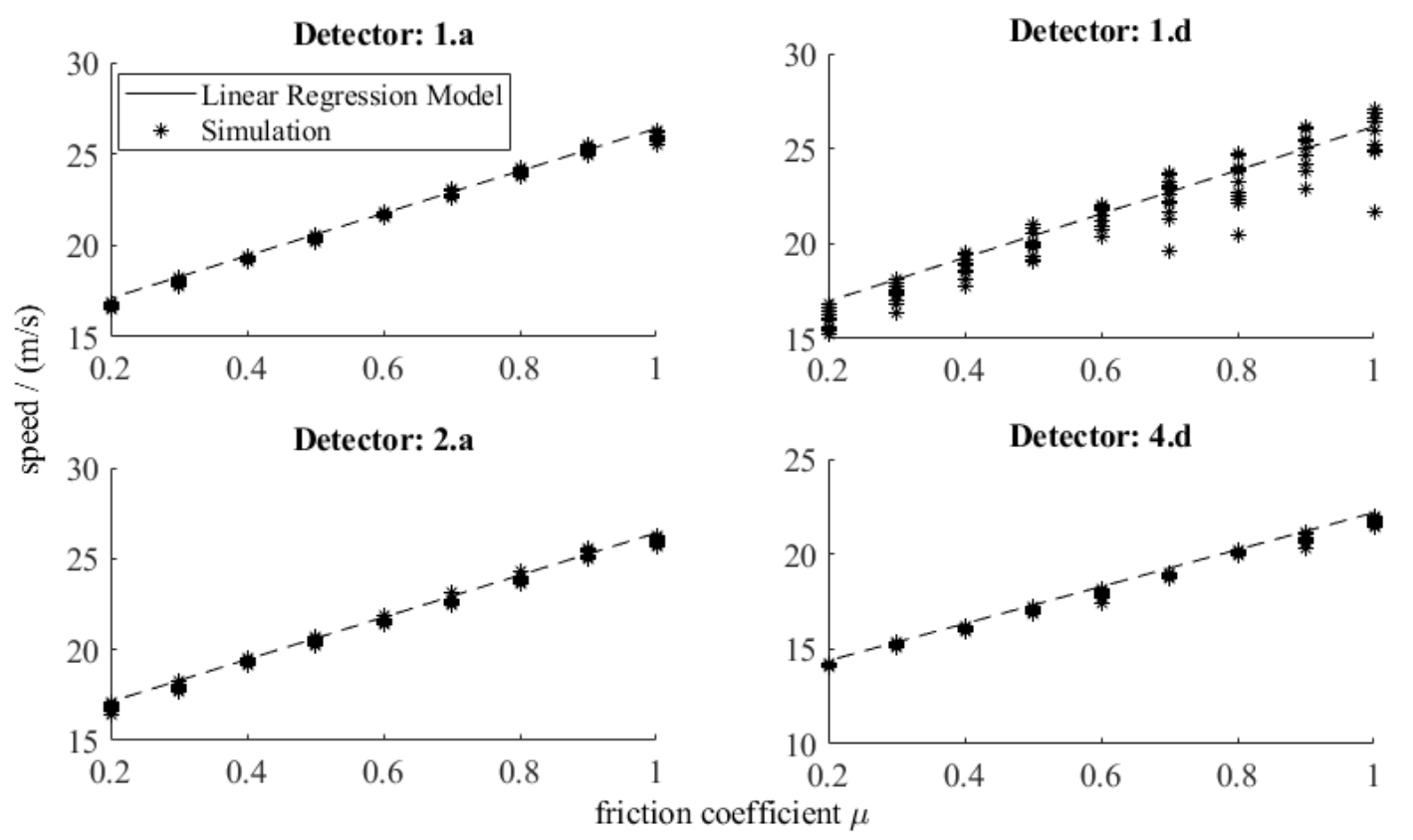

Figure 8: Effect of the linear adaptive car follow model

Table 3 shows the observed speed reductions within the described scenario using the linear model adapted version of the standard Krauss car following model for all available detector stations. When summarized, a speed reduction on wet road surface conditions of $0-23.5 \%$ is observed as well as a speed reduction range of 8.6-41.9 $\%$ on slippery road surface conditions. It is clear, that the inherent dynamic of the scenario leads to a wider spread of the range of speed reduction than expected from the use of the linear model depicted in Figure 5. 


\begin{tabular}{|l|l|l|l|l|l|l|l|l|}
\hline $\begin{array}{l}\text { Road } \\
\text { Condition }\end{array}$ & \multicolumn{7}{|c|}{ Detector Station } \\
\hline & $1 . \mathrm{a}$ & $1 . \mathrm{b}$ & $1 . \mathrm{c}$ & $1 . \mathrm{d}$ & $2 . \mathrm{a}$ & $2 . \mathrm{b}$ & $2 . \mathrm{c}$ & $2 . \mathrm{d}$ \\
\hline $\begin{array}{l}\text { Wet } \\
\text { (lower) }\end{array}$ & $19,1 \%$ & $19,8 \%$ & $19,3 \%$ & $23,5 \%$ & $19,1 \%$ & $22,6 \%$ & $21,7 \%$ & $19,4 \%$ \\
\hline $\begin{array}{l}\text { Slippery } \\
\text { (upper) }\end{array}$ & $13,9 \%$ & $14,4 \%$ & $14,5 \%$ & $13,7 \%$ & $14,5 \%$ & $8,6 \%$ & $13,7 \%$ & $14,1 \%$ \\
\hline $\begin{array}{l}\text { Slippery } \\
\text { (lower) }\end{array}$ & $32,4 \%$ & $30,5 \%$ & $32,3 \%$ & $35,2 \%$ & $32,3 \%$ & $35,7 \%$ & $41,9 \%$ & $31,6 \%$ \\
\hline
\end{tabular}

\begin{tabular}{|l|l|l|l|l|l|l|l|l|}
\cline { 2 - 8 } \multicolumn{1}{c|}{} & $3 . \mathrm{a}$ & $3 . \mathrm{b}$ & $3 . \mathrm{c}$ & $3 . \mathrm{d}$ & $4 . \mathrm{a}$ & $4 . \mathrm{b}$ & $4 . \mathrm{c}$ & $4 . \mathrm{d}$ \\
\hline $\begin{array}{l}\text { Wet } \\
\text { (lower) }\end{array}$ & $19,3 \%$ & $19,5 \%$ & $19,4 \%$ & $20,1 \%$ & $19,5 \%$ & $19,0 \%$ & $19.6 \%$ & $19,8 \%$ \\
\hline $\begin{array}{l}\text { Slippery } \\
\text { (upper) }\end{array}$ & $13,9 \%$ & $14,1 \%$ & $14,0 \%$ & $15,2 \%$ & $14,2 \%$ & $13,9 \%$ & $14,1 \%$ & $14,8 \%$ \\
\hline $\begin{array}{l}\text { Slippery } \\
\text { (lower) }\end{array}$ & $32,2 \%$ & $32,0 \%$ & $32,5 \%$ & $31,6 \%$ & $32,2 \%$ & $32,2 \%$ & $32,4 \%$ & $31,7 \%$ \\
\hline
\end{tabular}

Table 3: Observed max. relative speed reduction per detector station for the linear approach

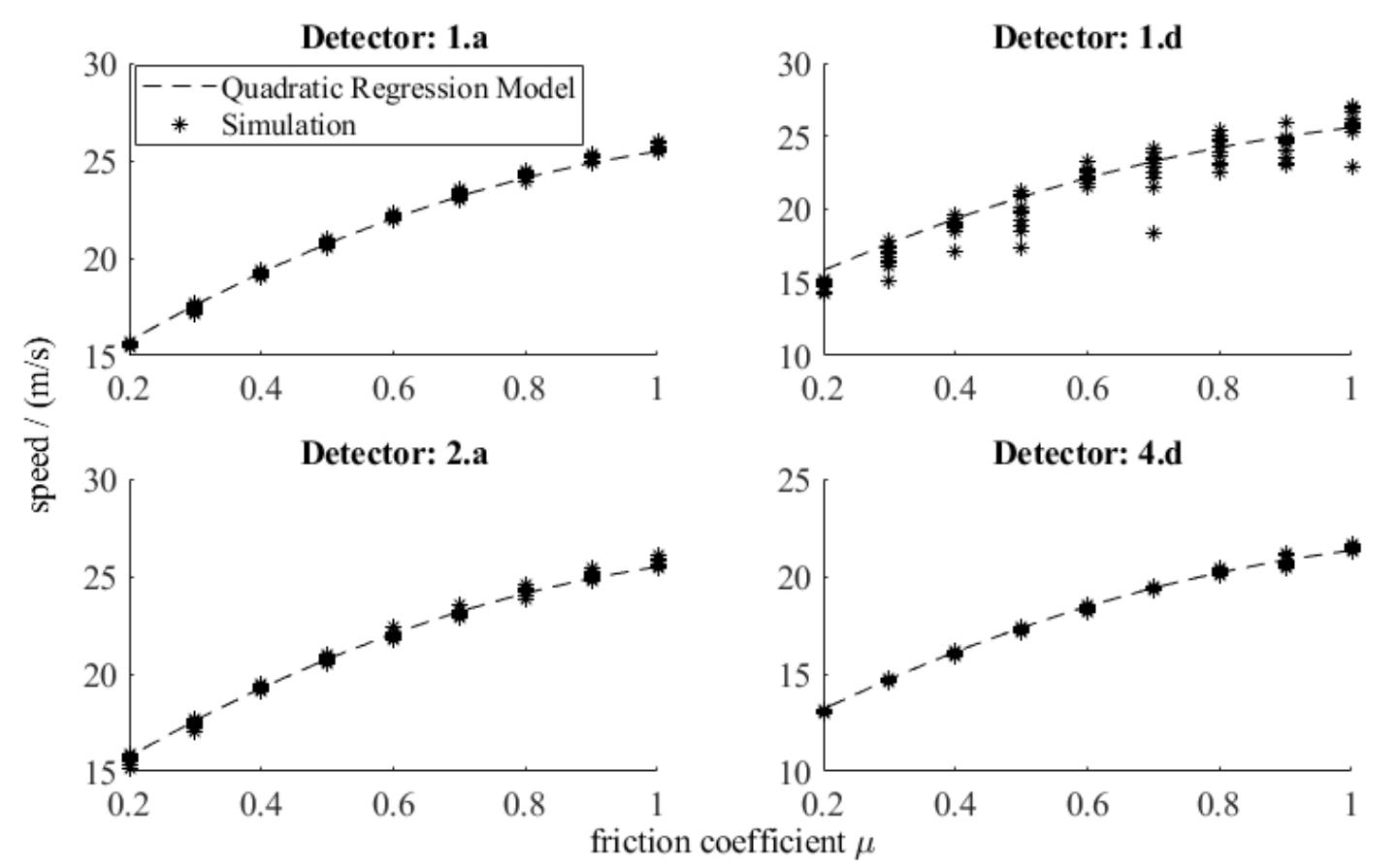

Figure 9: Effect of the quadratic adaptive car follow model 
In case of the quadratic adaptation according to equation (2-2) the observed reduction of average speeds follow the expectation very good, as can be taken from Figure 9. Overall a relative speed reduction up to $23.6 \%$ within the wet range and a reduction of $15.9 \%-38.8 \%$ within the snow equivalent slippery range is observed. It can be stated, that the simulated results of both implementations lay well within the limits of the behavior taken from the studies.

\begin{tabular}{|l|l|l|l|l|l|l|l|l|}
\begin{tabular}{|l|l|l|l|} 
Road \\
Condition
\end{tabular} & \multicolumn{7}{|c|}{ Detector Station } \\
\hline & $1 . \mathrm{a}$ & $1 . \mathrm{b}$ & $1 . \mathrm{c}$ & $1 . \mathrm{d}$ & $2 . \mathrm{a}$ & $2 . \mathrm{b}$ & $2 . \mathrm{c}$ & $2 . \mathrm{d}$ \\
\hline $\begin{array}{l}\text { Wet } \\
\text { (lower) }\end{array}$ & $21,3 \%$ & $21,4 \%$ & $21,1 \%$ & $22,2 \%$ & $20,9 \%$ & $21,7 \%$ & $23,6 \%$ & $22,0 \%$ \\
\hline $\begin{array}{l}\text { Slippery } \\
\text { (upper) }\end{array}$ & $16,3 \%$ & $16,5 \%$ & $16,8 \%$ & $16,5 \%$ & $16,7 \%$ & $15,9 \%$ & $17,4 \%$ & $16,9 \%$ \\
\hline $\begin{array}{l}\text { Slippery } \\
\text { (lower) }\end{array}$ & $30,6 \%$ & $29,6 \%$ & $30,7 \%$ & $32,2 \%$ & $30,8 \%$ & $27,9 \%$ & $38,3 \%$ & $30,3 \%$ \\
\hline & $3 . \mathrm{a}$ & $3 . \mathrm{b}$ & $3 . \mathrm{c}$ & $3 . \mathrm{d}$ & $4 . \mathrm{a}$ & $4 . \mathrm{b}$ & $4 . \mathrm{c}$ & $4 . \mathrm{d}$ \\
\hline $\begin{array}{l}\text { Wet } \\
\text { (lower) }\end{array}$ & $21,3 \%$ & $21,3 \%$ & $21,5 \%$ & $22,0 \%$ & $21,6 \%$ & $21,0 \%$ & $21,6 \%$ & $21,7 \%$ \\
\hline $\begin{array}{l}\text { Slippery } \\
\text { (upper) }\end{array}$ & $16,6 \%$ & $16,7 \%$ & $16,6 \%$ & $17,8 \%$ & $16,9 \%$ & $16,7 \%$ & $16,7 \%$ & $17,9 \%$ \\
\hline $\begin{array}{l}\text { Slippery } \\
\text { (lower) }\end{array}$ & $30,5 \%$ & $30,4 \%$ & $30,8 \%$ & $30,2 \%$ & $30,6 \%$ & $31,0 \%$ & $30,8 \%$ & $30,0 \%$ \\
\hline
\end{tabular}

Table 4: Observed max. rel. speed reduction per detector station for the quadratic approach

\section{Conclusion and Outlook}

This paper presents an implementation of road surface conditions into the microscopic traffic simulation framework SUMO. Firstly, it shows how the new attribute is introduced into the road network and the modules dealing with its creation and manipulation. It is then discussed how the new attribute is made accessible from the inside as well as the outside during a simulation. From the inside two main access points are implemented, directly through the elements of the network and through a device, allowing to simulate a sensor system. The first approach is used to adapt the car follow model in order to simulate how real drivers adapt to the surrounding conditions, the latter when using SUMO within a larger simulation environment that incorporates Car2X communication utilizing TraCI. To show the successful implementation of all intended elements, a real-world data calibrated example simulation is set up and in turn employed to show how the implemented road surface 
condition can be used to adapt the driving behavior in terms of chosen speeds as studies form the literature suggest. It turns out that the chosen models yield very good results that lay well within the limits of the findings from the various studies in terms of speed reduction with respect to wet and slippery road conditions.

Intended future works will include the creation of further relevant realistic traffic scenarios for urban and suburban areas, the implementation of additional road condition dependent systems such as a road friction dependent full range advanced cruise (FRACC) and an investigation of its impact on traffic flow as well as traffic safety characteristics. Another field of investigation based on this SUMO implementation will be in the realm of connected driving, such as the feasibility of distributing sensor data in an expected Car2X environment both via direct communication and through backend driven processing. The core of all these investigations will be authentic and calibrated situations and scenarios in SUMO such as the one presented in this paper to serve as a reference and base for comparison for the upcoming implementations.

\section{Literature}

[1] I. Weber, "Verbesserungspotenzial von Stabilisierungssystemen im Pkw durch eine Reibwertsensorik," Dissertation, Technische Universität Darmstadt, 2004.

[2] T. Tettamanti, I. Varga, and Z. Szalay, "Impacts of autonomous cars from a traffic engineering perspective," Periodica Polytechnica Transportation Engineering, vol. 44, pp. 244-250, 2016.

[3] BAST, "Technische Lieferbedingungen für Streckenstationen (TLS)," Bergisch Gladbach, 2002.

[4] T. Benz, F. Christen, G. Lerner, M. Schulze, and D. Vollmer, "Traffic effects of driver assistance systems-The approach within INVENT," 2003.

[5] P. Alvarez Lopez, M. Behrisch, L. Bieker-Walz, J. Erdmann, Y.-P. Flötteröd, R. Hilbrich, et al., "Microscopic Traffic Simulation using SUMO," presented at the The 21st IEEE International Conference on Intelligent Transportation Systems, Maui, USA, 2018.

[6] F. Klempau, "Untersuchungen zum Aufbau eines Reibwertvorhersagesystems im fahrenden Fahrzeug," Dissertation, Fachbereich Maschinenbau, Technische Universität Darmstadt, Darmstadt, 2003.

[7] C. Sommer, R. German, and F. Dressler, "Bidirectionally coupled network and road traffic simulation for improved IVC analysis," IEEE Transactions on Mobile Computing, vol. 10, pp. 3-15, 2011.

[8] A. Varga, "OMNeT++," in Modeling and tools for network simulation, ed: Springer, 2010, pp. 35-59. 
[9] M. Agarwal, T. H. Maze, and R. Souleyrette, "Impacts of weather on urban freeway traffic flow characteristics and facility capacity," in Proceedings of the 2005 mid-continent transportation research symposium, 2005, pp. 18-19.

[10] A. D. Stern, V. Shah, L. Goodwin, and P. Pisano, "Analysis of weather impacts on traffic flow in metropolitan Washington DC," in Proceedings of the 19th International Conference on Interactive Information and Processing Systems (IIPS) for Meteorology, Oceanography, and Hydrology, 2003.

[11] HCM, "Highway Capacity Manual," Third ed. Washington DC: Transport Research Board, 2000.

[12] L. C. Goodwin, "Weather impacts on arterial traffic flow," Mitretek systems inc, 2002.

[13] S. Krauß, "Microscopic modeling of traffic flow: Investigation of collision free vehicle dynamics," Universitat zu Koln., 1998.

[14] C. Gerhard and I. Schneidermann. (2018, 18.12.2018). Automatische Zählstellen auf Autobahnen und Bundesstraßen. Available: https://www.bast.de/BASt_2017/ DE/Verkehrstechnik/Fachthemen/v2verkehrszaehlung/Stundenwerte.html

[15] D. Kraft, "A software package for sequential quadratic programming," Forschungsbericht- Deutsche Forschungs- und Versuchsanstalt fur Luft- und Raumfahrt, 1988. 